\title{
Primary intraorbital extraocular primitive neuroectodermal (neuroepithelial) tumour
}

\author{
SAMRUAY SHUANGSHOTI, ' WIT MENAKANIT, ${ }^{2}$ WITIT CHANGWAIVIT,${ }^{3}$ AND \\ NITA YA SUWANWELA+ \\ From the 'Department of Pathology, Faculty of Medicine, Chulalongkorn University, Bangkok; the \\ ${ }^{2}$ Department of Pathology, Faculty of Medicine, Chiang Mai University, Chiang Mai; the ${ }^{3}$ Department of \\ Radiology, Faculty of Medicine, Chiang Mai University, Chiang Mai; and the ${ }^{4}$ Department of Radiology, \\ Faculty of Medicine, Chulalongkorn University, Bangkok, Thailand
}

SUMMARY A case is reported of primary primitive neuroectodermal (neuroepithelial) tumour occurring in the right orbit of a 52-year-old man. The intraorbital extraocular location is unique for this kind of neoplasm. The malignant tumour was differentiated into primitive neuroepithelial, ependymal, and oligodendroglial cells. The neuroglia was identified by localisation of cytoplasmic glial fibrillary acidic protein. It is suggested that this primary intraorbital, extraocular, primitive neuroectodermal tumour with neuroglial differentiation is akin to the primitive neuroectodermal tumours of the neuraxis, including the cerebellar medulloblastomas, and to some peripheral nerve tumours known as malignant neuroepitheliomas, malignant ependymomas, and neuroblastomas. The ectomesenchymal remnant of the neural crest or ectopic neuroepithelium or both may have been the origin of the present tumour. Differentiation of the neuroectodermal component of the neural crest or heterotopic neuroepithelium or both would create a primitive neuroectodermal tumour with diverse neuroglial elements.

Primitive neuroectodermal tumours of the neuraxis ${ }^{1 \cdot 3}$ and peripheral nerves ${ }^{45}$ have been described. However, we are unable to trace a report of the same type of tumour arising primarily within the orbit but outside the eyeball. In this communication we describe a primary intraorbital, extraocular, primitive neuroectodermal tumour occurring in an adult man.

\section{Case report}

A 52-year-old man was admitted to hospital because of progressive right exophthalmos for one month. He denied ocular pain, blurred vision, diplopia, and other systemic symptoms.

Physical examination revealed a body temperature of $37^{\circ} \mathrm{C}$, pulse rate 85 beats/min, respiratory rate $20 / \mathrm{min}$, and blood pressure $150 / 90 \mathrm{mmHg}$. The right eye showed mild oedema of the eyelids and proptosis. Each pupil, $3 \mathrm{~mm}$ in diameter, was reactive to light. Ocular movements were bilaterally full in all directions. The fundi showed constriction of the

Correspondence to Professor Samruay Shuangshoti, Department of Pathology, Chulalongkorn Hospital, Bangkok 10500, Thailand. arterioles. No papilloedema or intraocular tumour was observed. Cranial nerves, visual field, and visual acuity were normal, as were other systems of the body and various laboratory tests. No abnormal masses were detected in any part of his body.

Computerised tomographic (CT) scans of the orbits and brain disclosed a right enlarging intraorbital tumour located laterally. The right eyeball and optic nerve were displaced medially (Figs. 1,2). No intracranial lesions were seen.

The patient underwent Krönlein's operation three weeks after admission to hospital. The outer wall of the right orbit was resected. The laterally situated intraorbital extraocular tumour was found to be unencapsulated and attached to the lateral rectus muscle. There was no bone invasion. The lesion was removed as much as possible. No connection between the tumour and any nerve was observed. It appeared clinically to be a neoplasm of the lacrimal gland.

PATHOLOGICALEXAMINATION

The formalin-fixed tissue fragment was white, firm, and $3.5 \times 4.0 \times 4.0 \mathrm{~cm}$. It was embedded in paraffin 

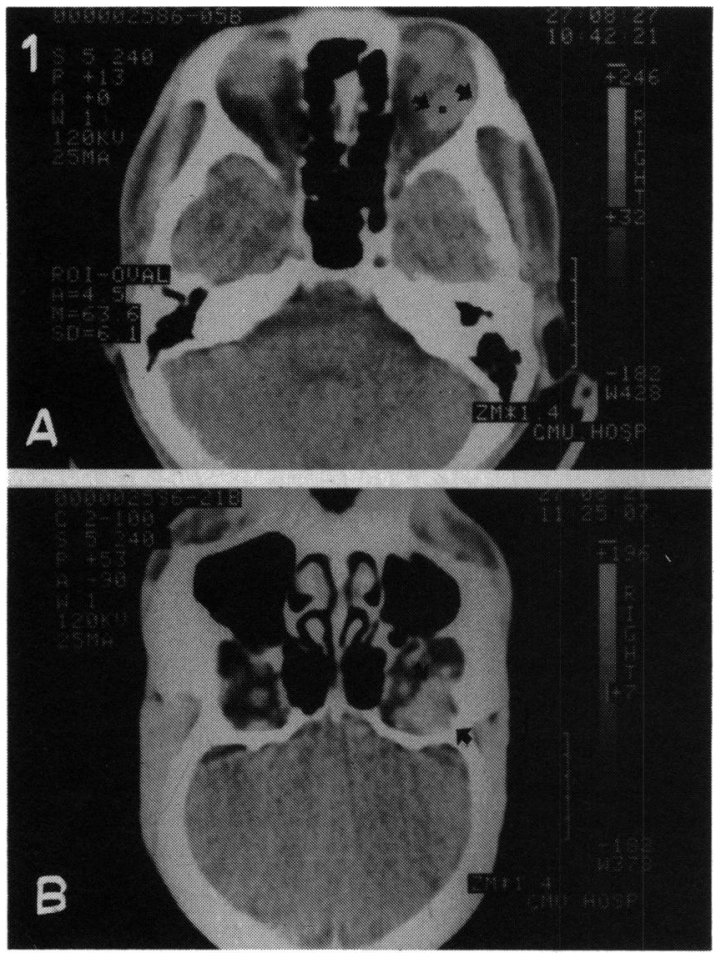

Fig. 1 A, B Axial and coronal CT scans of the orbits show an infiltrative soft tissue mass in the superolateral part of the right orbit (left arrows) with pressure effect on the lateral wall (right arrows).

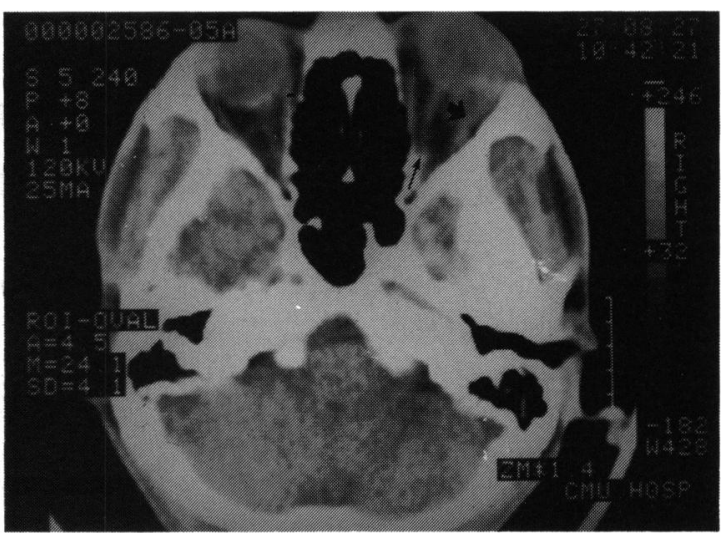

Fig. 2 Axial cut at the level of the optic nerves demonstrates a mass invading into the right lateral rectus muscle (thick arrow). Note also medial displacement of the right optic nerve (thin arrow) and mild proptosis of the right eyeball.

and sections were stained with haematoxylin and eosin (H-E). The following special stains were done: Mallory's phosphotungstic acid haematoxylin (PTAH) for neuroglial fibres, Gomori's stain for reticulin fibres, and Nissl's stain for neuronal tigroid substance.

The peroxidase-antiperoxidase technique was applied for immunohistochemical localisation of glial fibrillary acidic protein (GFAP) in formalin-fixed and paraffin-embedded tissue sections. A fragment of the normal brain was similarly processed as a control.
Fig. 3 Microscopic features of tumour. A: Numerous small neoplastic cells in lobules and in no definite pattern of arrangement are shown $(H-E, \times 32)$. B: Homer Wright's rosettes are formed by primitive neuroepithelial tumour cells. Note tangles of cellular processes within the centre of rosettes $(H-E, \times 65)$.
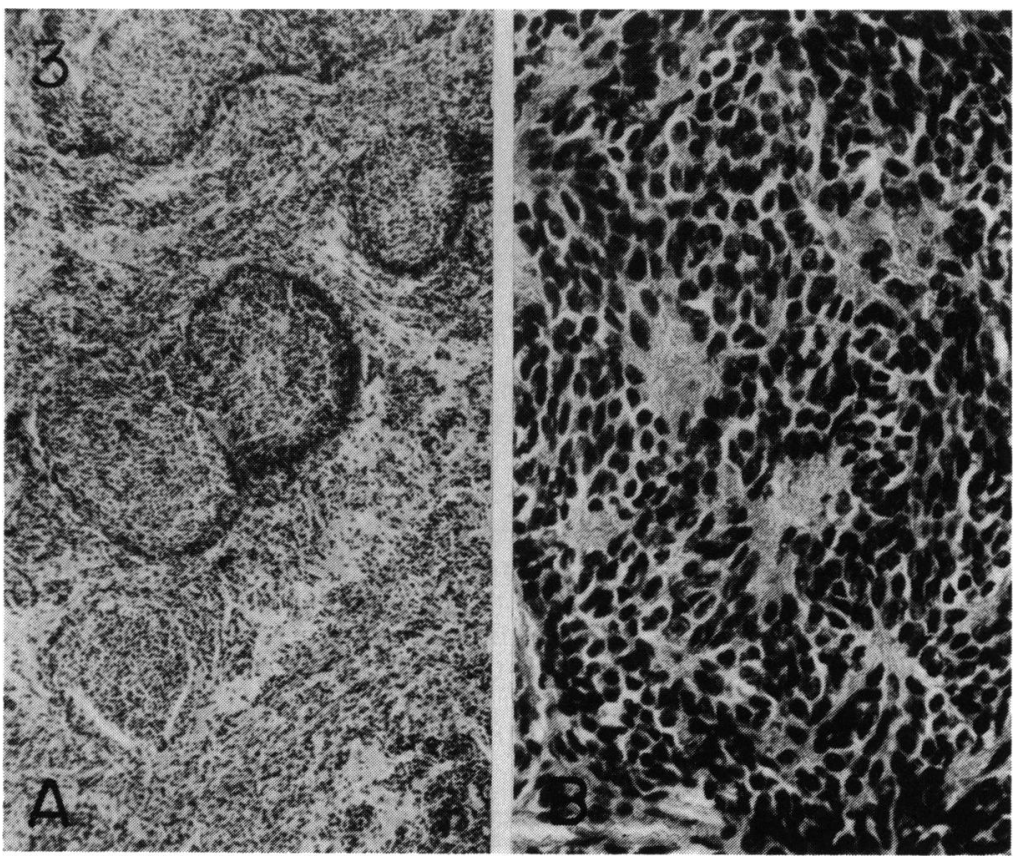
Microscopically, plentiful neoplastic cells of divergent types were found in lobules and in random distribution (Fig. 3A). Many small ovoid or carrotshaped tumour cells with hyperchromatic nuclei rimmed by scant cytoplasm and hair-like cytoplasmic polar processes were often arrayed in rosettes that contained central tangles of these processes, representing Homer Wright's rosettes (Fig. 3B). These neoplastic cells were regarded as primitive (undifferentiated) neuroepithelial cells which resembled both neuroblasts and medulloblasts.

Several epithelium-like tumour cells had round to ovoid vesicular nuclei which were rimmed by homogeneous cytoplasm. Some had blue processes in PTAH preparations. They were occasionally arranged in true rosettes with central canals (Fig. 4A) and perivascular pseudorosettes characterised by attachment of the cellular processes to the vascular walls (Fig. 4B). These two patterns of the neoplasm were regarded as formed by ependymal cells.

A moderate number of the tumour cells had well defined outlines, distinctly clear perikaryon, and small dark nuclei giving a fried-egg appearance. They were interpreted as oligodendroglia (Figs. 4B, 5A). Neurons were not detected in Nissl's preparations.

A small number of widely scattered rounded tumour cells contained cytoplasmic GFAP and were regarded as neuroglia. Some had small dark nuclei surrounded by moderate amount of cytoplasm; they were interpreted as oligodendroglia (Fig. 5B). Others had large hyperchromatic nuclei rimmed by scant perikaryon; they were regarded as undiffer- entiated neuroglia (Fig. 5C). Similar GFAP-positive neuroglia, particularly astrocytes, were also seen in the fragment of normal brain serving as a control.

Reticulin fibres were few in some parts of the tumour but were numerous elsewhere (Fig. 6). This was also true for collagen fibres.

The pathological diagnosis was primary intraorbital, extraocular, primitive neuroectodermal tumour showing neuroglial differentiation and producing right proptosis.

\section{POSTOPERATIVE COURSE}

The patient received a course of radiotherapy totalling $5200 \mathrm{rad}$ to the operative field over $1 \frac{1}{2} 2$ months. At the end of the radiotherapeutic course his vision was normal, as it was before the surgical intervention. The proptosis had improved, and the general condition was satisfactory. He was well when he was seen again 6 months later.

\section{Discussion}

The primitive neuroectodermal tumour is defined as a malignant neoplasm arising from primitive (stem cell) neuroepithelium which is capable of differentiating into various derivatives within the neuronal and neuroglial lines. Pathologically, Homer Wright's rosettes formed by primitive neuroepithelial cells are present. True rosettes and perivascular pseudorosettes may be present. The tumour may show a prominent mesenchymal component. ${ }^{13}$

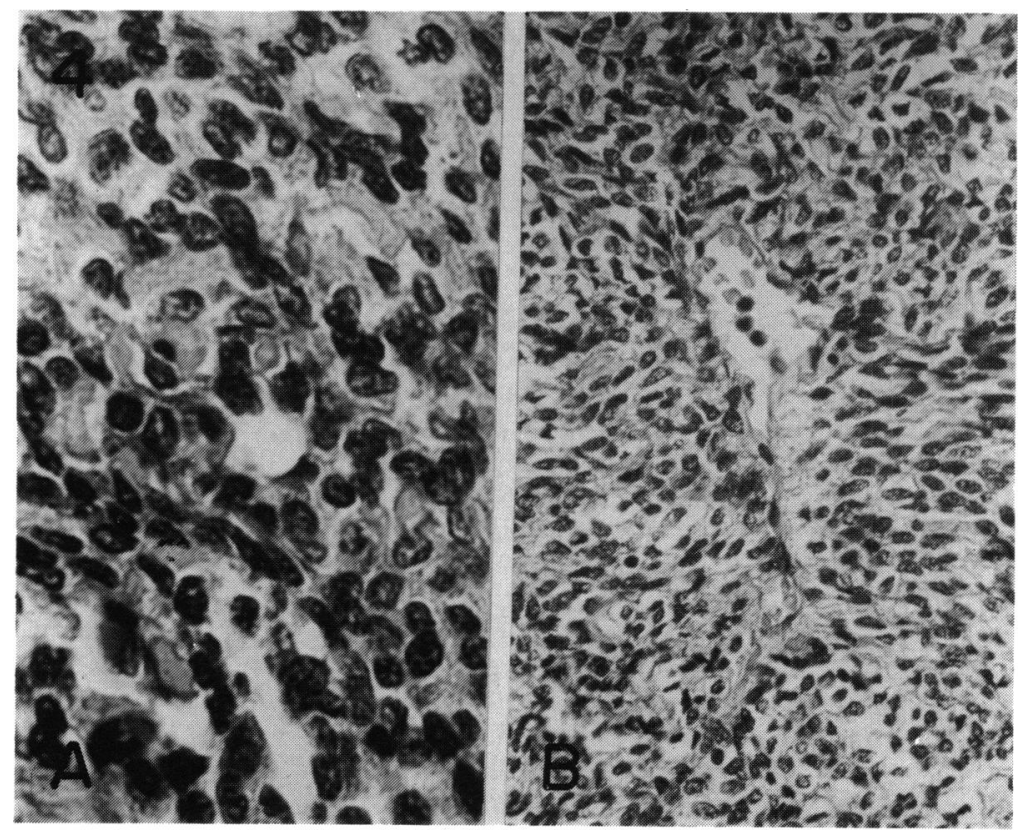

Fig. 4 Ependymal and oligodendroglial differentiation of tumour. A: A true rosette formed by radially arranged ependymal tumour cells round central canal is shown $(H-E, \times 253)$. B: $A$ perivascular pseudorosette consisting of central blood vessel with processes of ependymal tumour cells attached to its wall is shown. Some rounded neoplastic cells having distinctly clear perikaryon round small nuclei to give fried-egg appearance, as in a cluster at the right lower corner of the photomicrograph, are considered to be oligodendroglia (H-E, × 63). 

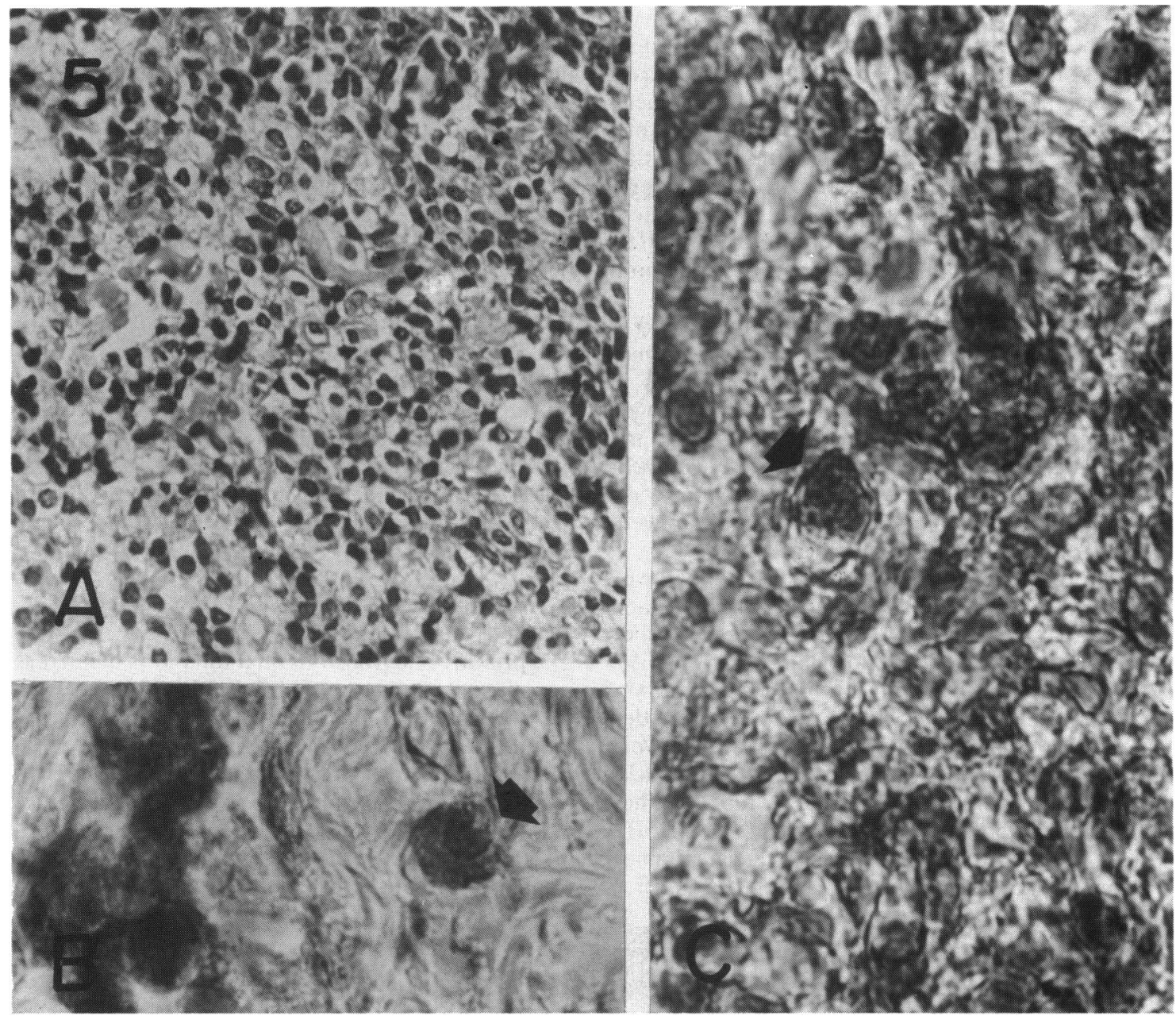

Fig.5 Oligodendroglial differentiation and result of immunohistochemical study. A: Many oligodendroglia with fried-egg appearance are shown $(H-E, \times 100)$. B: $A$ GFAP-positive tumour cell interpreted as oligodendrocyte $($ arrow $)$ is demonstrated (GFAP stain, $\times 1000)$. C: A neoplastic cell having large hyperchromatic nucleus surrounded by scant $G F A P$-positive perikaryon regarded as undifferentiated neuroglial cell (arrow) is present among other undifferentiated tumour cells (GFAPstain, $\times 1000)$.

The intraorbital extraocular neoplasm described here fulfils these criteria for designating it as a primitive neuroectodermal tumour because it had primitive neuroepithelial cells forming Homer Wright's rosettes, ependymal cells forming true rosettes and perivascular pseudorosettes, and neoplastic oligodendroglia. The present of neuroglia within the tumour was confirmed by the localisation of cytoplasmic GFAP in some tumour cells, as we have demonstrated previously..$^{6.4}$ In those studies all kinds of neuroglia (astrocytes, ependymal cells, oligodendroglia, and undifferentiated neuroglia) were GFAP-positive..$^{6.4}$

Nevertheless, the presence of primitive neuro- epithelial cells forming Homer Wright's rosettes in the present tumour suggests that it is a neuroblastoma. Indeed, neuroblastomas in adult patients have been reported." However, the concept of the primitive neuroectodermal tumour encompasses as well the neuroblastomas. A neuroblastoma can be perceived as primitive neuroectodermal tumour with entire neuroblastic differentiation. The present intraorbital extraocular neoplasm is a primitive neuroectodermal tumour with neuroglial differentiation. Nevertheless a small number of GFAP-positive neuroglial neoplastic cells as well as prominent reticulin and collagen fibres in some parts of this tumour may not support the diagnosis of the primitive 

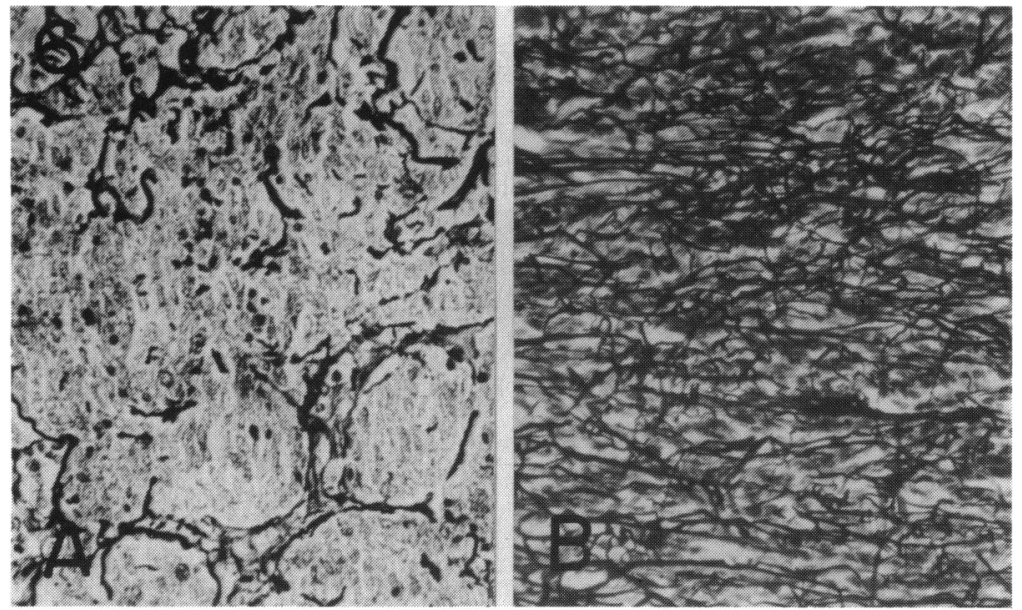

Fig. 6 Reticulin fibres within tumour. A: Few reticulin fibres are present around some lobules of the tumour cells but are absent within lobules (Gomori's stain, × 72). B: Dense reticulin fibres are shown in a field of less numerous neoplastic cells (Gomori's stain, $\times 72$ ). neuroectodermal tumour but favour a mesenchymal origin of the neoplasm. According to our experience, however, ependymal and oligodendroglial tumour cells are not frequently GFAP-positive, as are astrocytes, which were not seen in the present tumour. A prominent mesenchymal component is known in primitive neuroectodermal neoplasms.'

We regard the current primary intraorbital, extraocular, primitive neuroectodermal tumour as akin to those neoplasms reported as neuraxial primitive neuroectodermal tumours, including the cerebellar medulloblastomas ${ }^{1-311}$ and to some peripheral nerve neoplasms which have been described as malignant neuroepitheliomas, ${ }^{12-14}$ malignant ependymoma, ${ }^{15}$ and primitive neuroectodermal tumour (neuroblastoma). ${ }^{5}$ These peripheral nerve tumours contained Homer Wright's rosettes and true rosettes as in our patient's tumour relating to the orbit. They often metastasised widely. ${ }^{52-15}$

The primitive (stem cell) neuroepithelium has been suggested as the origin of the neuraxial primitive neuroctodermal tumours. ${ }^{311}$ For the peripheral primitive neuroectodermal tumours an origin from the neural crest has been proposed. ${ }^{9}$ Evidence has been presented relating to the combined mesenchymal and neuroectodermal (ectomesenchymal) nature of the neural crest, ${ }^{9}{ }^{16-1 x}$ because it is formed by mingling of the mesenchyme and developing neuroepithelium of the neural folds before closure of the neural tube. ${ }^{17}$ The neural crest yields diverse derivatives of both mesenchymal and neuroepithelial lines which migrate throughout the body, particularly in association with the peripheral nerves. ${ }^{14}$ Although no connection of the present tumour with any major nerve was observed clinically, its origin from a small nerve, such as a nerve twig, cannot be excluded. Moreover, in the development of the eyeball, which has been reviewed previously,,$^{21}$ the primitive neuro- epithelium forming the optic primordium may become ectopic within the orbit, a comparable developmental anomaly known as heterotopia of the neuraxis. $^{81}$ This heterotopic neural tissue and/or neuroectodermal component of the neural crest comprising the sheath of the peripheral nerve within the right orbit may be the origin of our patients tumour, which further differentiated into diverse neuroepithelial derivatives.

Professor Samruay Shuangshoti is in receipt of funds from the Anandhamahidol Foundation, Medical Section, Bangkok, Thailand.

\section{References}

1 Hart MN, Earle K. Primitive neuroectodermal tumours of the brain in children. Cancer 1973; 32: 890-7.

2 Kosnick EJ, Boesel CP, Bay J, Sayers MP. Primitive neuroectodermal tumours of the central nervous system in children. J Neurosurg 1978; 48: 741-6.

3 Rorke LB. The cerebellar medulloblastoma and its relationship to primitive neuroectodermal tumours. J Neuropathol Exp Neurol 1983; 42: 1-15

4 Seemayer TA, Thelmo WL, Bolande RP, Wiglesworth FW. Peripheral neuroectodermal tumours. Perspect Pediatr Pathol $1975 ; 2: 151-72$.

5 Nesbitt KA, Vidone RA. Primitive neuroectodermal tumour (neuroblastoma) arising in sciatic nerve of a child. Cancer 1976; 37: $1562-70$.

6 Shuangshoti S, O'Charoen S. Cerebellar neoplasm of mixed mesenchymal and neuroepithelial origin: case report. J Neurosurg 1983; 59: 337-43

7 Shuangshoti S, Suwanwela N, Suwanwela C. Combined occurrence of paraganglioma and glioma of conus medullaris and cauda equina. J Surg Oncol 1984; 25: 162-7.

8 Shuangshoti S, Kasantikul V, Suwanwela N, Suwanwela C Solitary intracranial extracerebral gliomas: case report. J Neurosurg 1984; 61: 777-81.

9 Shuangshoti S, Kasantikul V, Suwangool P, Chittmittrapap S. Malignant neoplasm of mixed mesenchymal and neurocpithelial origin (ectomesenchymoma) of thigh. J Surg Oncol 1984; 27: 208-13.

10 Mackay B, Luna MA, Butler JJ. Adult neuroblastoma: electron microscopic observations in nine cases. Cancer 1976; 37: $1334-51$. 
11 Palmer JO, Kasselberg AG, Netsky MG. Differentiation of medulloblastoma: studies including immunohistochemical localisation of glial fibrillary acidic protcin. J Neurosurg 1981; 55: $161-9$.

12 Stout AP. A tumour of the ulnar nerve. Proc $N$ Y Pathol Soc 1918; 18: 2-11.

13 Lagerkvist B, Ivemark B, Sylven B. Malignant neuroepithelioma in children: a report of threc cases. Acta Chir Scand 1969; 135: 641-5.

14 Bolen JW, Thorning D. Peripheral neurocpithelioma: A light and electron microscopic study. Cancer 1980; 46: 2456-62.

15 Lanford JA, Cohen I. Ependymal neoplasm of median nerve with case report. South Med J 1927; 20: 273-8.

16 Shuangshoti S, Chongchet V. Malignant mesenchymoma of ulnar nerve: combined sarcoma of nerve sheath and rhabdomyosarcoma. J Neurol Neurosurg Psychiatry 1979; 42: 524-8.
17 Shuangshoti S. Mclanotic mucin-producing ncurocpithclial ncoplasm of mesencephalon with consideration of similar tumours in different locations. J Neurol Neurosurg Psychiatry 1980; 43: $810-7$.

18 Shuangshoti S, Benjavongkulchai S, Chittmittrapap S. Malignant mesenchymoma of median nerve: combined nerve sheath sarcoma and liposarcoma. J Surg Oncol 1884; 25: 119-23.

19 Weston TA. The migration and differentiation of neural crest cell. In: Abercrombic M, Brachet J, King TJ. eds. Advances in morphogenesis. New York: Academic Press, 1970): 41-114.

20 Shuangshoti S. Meningioma of the optic nerve. BrJ Ophthalmol 1973; 57: 265-9.

21 Shuangshoti S, Yenrudi S. Netsky MG. Heterotopias of brain as a cause of infantile regurgitation. Teratology 1981; 23: 67-73.

Accepted for publication 2I November 1985. 